\title{
Prognostic and therapeutic implications of mTORC1 and Rictor expression in human breast cancer
}

\author{
U. WAZIR ${ }^{1,2}$, R.F. NEWBOLD ${ }^{3}$, W.G. JIANG ${ }^{4}$, A.K. SHARMA ${ }^{1}$ and K. MOKBEL ${ }^{1,2}$ \\ ${ }^{1}$ The London Breast Institute, Princess Grace Hospital, London; ${ }^{2}$ Department of Breast Surgery, St. George's Hospital \\ and Medical School, University of London, London; ${ }^{3}$ Brunel Institute of Cancer Genetics and Pharmacogenomics, \\ Faculty of Life Sciences, Brunel University, Uxbridge, London; ${ }^{4}$ Metastasis and Angiogenesis Research Group, \\ University Department of Surgery, Cardiff University School of Medicine, Cardiff University, Cardiff, Wales, UK
}

Received October 3, 2012; Accepted November 12, 2012

DOI: $10.3892 /$ or.2013.2346

\begin{abstract}
The mammalian target of rapamycin (mTOR) plays a key role in the regulation of cellular metabolism, growth and proliferation. It forms two multi-protein complexes known as complex 1 (mTORC1) and 2 (mTORC2). Raptor and Rictor are the core proteins for mTORC1 and mTORC2, respectively. This study examines the relationship between mTORC1, Rictor and Raptor mRNA expression and human breast cancer. Furthermore, the correlation between mTORC1 and hTERT was investigated. Breast cancer tissues $(n=150)$ and normal tissues $(n=31)$ were analysed using reverse transcription and quantitative PCR. Transcript levels were correlated with clinicopathological data. Higher mTOR expression was noted in breast cancer tissue $(\mathrm{P}=0.0018)$, higher grade tumours (grade 2 vs. $3, \mathrm{P}=0.047)$, in ductal tumours $(\mathrm{P}=0.0014)$, and was associated with worse overall survival $(\mathrm{P}=0.01)$. Rictor expression was significantly higher in background breast tissues compared with tumours and was inversely related to the Nottingham Prognostic Index (NPI1 vs. 2, P=0.03) and tumour grade (grade 1 vs. $3, \mathrm{P}=0.01$ ) and was associated with better overall $(\mathrm{P}=0.037)$ and disease-free survival $(\mathrm{P}=0.048)$. The mRNA expression of Raptor was higher in tumours compared with normal tissues. Furthermore, the expression of Raptor was associated with a higher tumour grade (grade 1 vs. $3, \mathrm{P}=0.027)$. A highly significant positive correlation between $\mathrm{mTOR}$ and hTERT $(\mathrm{P}<0.00001)$ was observed. These observations are consistent with the role of mTORC1 in the anti-apoptosis pathway and suggest that selective inhibitors of mTORC1 may be more efficacious in human breast cancer. Our findings support the hypothesis that mTORC1 is an important upregulator of telomerase in breast cancer.
\end{abstract}

Correspondence to: Professor Kefah Mokbel, The London Breast Institute, Princess Grace Hospital, 45 Nottingham Place, London W1U 5NY, UK

E-mail: kefahmokbel@hotmail.com

Key words: rapamycin, autophagy, qPCR, breast cancer, hTERT, telomerase, Rictor, Rheb, Raptor

\section{Introduction}

The mammalian target of rapamycin (mTOR) plays a key role in the regulation of cellular metabolism, growth and proliferation. It was found to mediate the anti-proliferative activities of rapamycin and its analogues (rapalogues). It forms two multi-protein complexes known as complex 1 (mTORC1) and 2 (mTORC2). Raptor and Rictor are the core proteins for $\mathrm{mTORC1}$ and $\mathrm{mTORC} 2$, respectively, known to be essential for the integrity of their respective complexes $(1,2)$. Rheb (Ras homologue enriched in brain) is a key activator of mTORC1. There is a growing body of evidence that mTORC1 is upregulated in many types of cancers and plays a role in carcinogenesis (3).

Rapalogues have been clinically proven in the case of renal cell carcinoma $(4,5)$. However, this success is yet to be replicated in the case of other cancers. A greater understanding of the role of mTOR in various types of cancers is needed to determine therapeutic strategies. To this end, studies have been carried out to identify potential markers of rapamycin sensitivity, as well as additional therapeutic agents $(6,7)$.

The aim of the study was to investigate the mRNA expression of mTORC1, Rictor, Raptor and Rheb in human breast cancer and examine the relationship between their expression and clinicopathological parameters. Furthermore, the correlation between mTORC1 and human telomerase reverse transcriptase (hTERT; the catalytic subunit of telomerase) was investigated.

\section{Materials and methods}

Samples. Tissue samples were collected after informed consent with ethical approval as per contemporaneous institutional guidelines. Immediately after surgical excision, a tumour sample was taken from the tumour area, while another was taken from the associated non-cancerous tissue (ANCT) within $2 \mathrm{~cm}$ of the tumour, without affecting the assessment of tumour margins. Breast cancer tissues $(n=150)$ and normal background tissues $(n=31)$ were collected and stored at $-80^{\circ} \mathrm{C}$ in liquid nitrogen until the commencement of this study.

All the patients were treated according to local guidelines, following discussions in multidisciplinary meetings. 
Table I. Clinical data describing the patient cohort.

\begin{tabular}{|c|c|}
\hline Parameter & No. of patients \\
\hline \multicolumn{2}{|l|}{ Node status } \\
\hline Node positive & 65 \\
\hline Node negative & 55 \\
\hline \multicolumn{2}{|l|}{ Tumour grade } \\
\hline 1 & 23 \\
\hline 2 & 41 \\
\hline 3 & 56 \\
\hline \multicolumn{2}{|l|}{ Tumour type } \\
\hline Ductal & 88 \\
\hline Lobular & 14 \\
\hline Medullary & 2 \\
\hline Tubular & 2 \\
\hline Mucinous & 4 \\
\hline Other & 4 \\
\hline \multicolumn{2}{|l|}{ TNM staging } \\
\hline 1 & 69 \\
\hline 2 & 40 \\
\hline 3 & 7 \\
\hline 4 & 4 \\
\hline \multicolumn{2}{|l|}{ Clinical outcome } \\
\hline Disease-free & 81 \\
\hline With local recurrence & 5 \\
\hline Alive with metastasis & 7 \\
\hline Succumbed to breast cancer & 20 \\
\hline
\end{tabular}

Patients undergoing breast-conserving surgery also underwent radiotherapy. Hormone-sensitive patients received tamoxifen. Hormone-insensitive cases, high-grade cancer, and node-positive cases were treated with adjuvant therapy. Clinicopathological data (Table I) were collected from the patient charts and collated in an encrypted database.

RNA extraction kits and reverse transcription kits were obtained from AbGene, Ltd. (Surrey, UK). PCR primers were designed using Beacon Designer (Premier Biosoft International, Ltd., Palo Alto, CA, USA) and synthesized by Invitrogen, Ltd. (Paisley, UK). Custom made Hot Start Master Mix for quantitative PCR was from AbGene (8).

Tissue processing, RNA extraction and cDNA synthesis. Approximately $10 \mathrm{mg}$ of cancerous tissue was homogenised. A larger amount of ANCT (20-50 mg) was used as its high fat content made it difficult to obtain sufficient RNA for analysis. The concentration of RNA was determined using a UV spectrophotometer (Wolf Laboratories, York, UK) to ensure adequate amounts of RNA for analysis. Reverse transcription was carried out using a reverse transcription kit (AbGene) with an anchored oligo(dT) primer using $1 \mathrm{mg}$ of total RNA in a 96-well plate to produce cDNA. The quality of cDNA was verified using $\beta$-actin primers (5'-ATGATATCGCCGCGCTCGTC-3' and 5'-CGCTCGGTGAGGATCTTCA-3') (8).

Quantitative analysis. Transcripts of the cDNA library were determined using real-time quantitative polymerase chain reaction (qPCR) based on Amplifluor technology. The PCR primers were designed using Beacon Designer software, but an additional sequence, known as the $\mathrm{Z}$ sequence (5'-ACTGAACCTGACCGTACA-3'), which is complementary to the universal $\mathrm{Z}$ probe (Intergen, Inc., Oxford, UK) was added to the primer. The primers used are detailed in Table II.

The reaction was carried out under the following conditions: $94^{\circ} \mathrm{C}$ for $12 \mathrm{~min}$ and 50 cycles of $94^{\circ} \mathrm{C}$ for $15 \mathrm{sec}, 55^{\circ} \mathrm{C}$ for $40 \mathrm{sec}$ and $72^{\circ} \mathrm{C}$ for $20 \mathrm{sec}$. The levels of each transcript were generated from a standard plasmid which contained the specific DNA sequence that was simultaneously amplified within the samples. With every run of the PCR, a negative and positive control was employed, using a known cDNA sequence (8).

Statistical analysis. Analysis of the data was performed using the Minitab 12 statistical software package (Minitab, Ltd., Coventry, UK) using a custom-written macro (Stat06e.mtb). Medians were compared using the Mann-Whitney U-test, while the means were compared using the two-sample t-test. The transcript levels within the breast cancer specimens were compared to those of the ANCT and correlated with clinicopathological data collected over a 10 -year follow-up period.

Table II. Primers used in the RT-PCR analysis.

Gene

Primer sequence

\section{mTORF1}

mTORZr1

RHEBF1

RHEBZr1

RaptorF1

RaptorZr1

RictorF1

RictorZr1

$\beta$-actin forward

$\beta$-actin reverse
CTGCAGAAGAAGGTCACT

ACTGAACCTGACCGTACAAAGGAGATGGAACGGAAG

TTGGTTGGGAATAAGAAAGA

ACTGAACCTGACCGTACAAAAAGCTGCATTCCAAGAT

TGAACACCGGACCATGAC

ACTGAACCTGACCGTACACAATGAGGTTTCCCTGAAG

AACTTGCAAAACAGTGTGAA

ACTGAACCTGACCGTACAATATCACAGCCTTGTTTGGT

ATGATATCGCCGCGCTCGTC

CGCTCGGTGAGGATCTTCA 


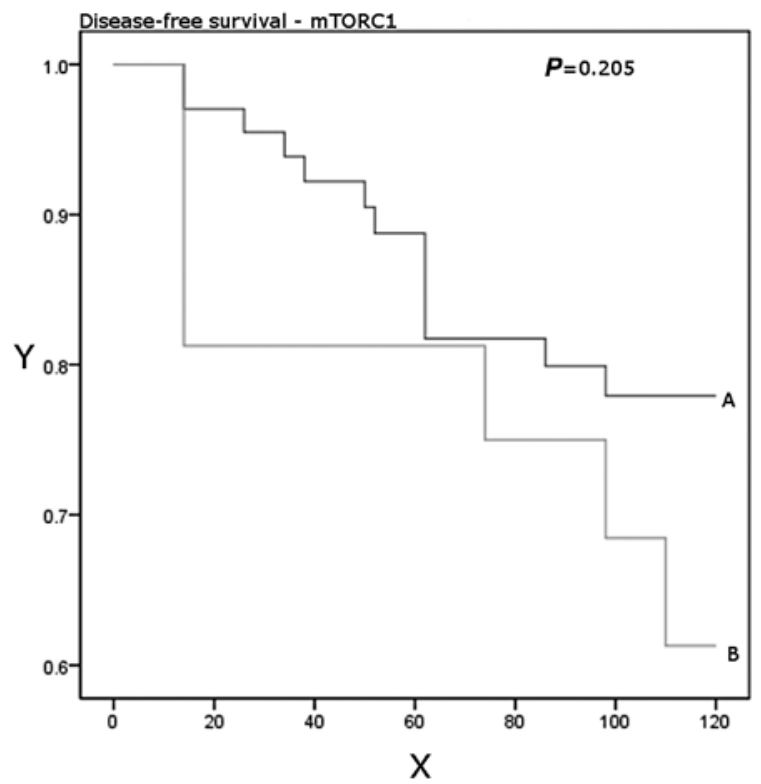

Figure 1. Disease-free survival curve for mTORC1. Curve A, lower transcription group as determined by moderate prognostic group as defined by NPI serving as the dividing line; Curve B, higher transcription group as determined by moderate prognostic group as defined by NPI serving as the dividing line; $\mathrm{X}$-axis, follow-up in months; Y-axis, cumulative survival.

P-values $<0.05$ were considered significant, whereas P-values between 0.05 and 0.10 were considered marginally significant. Correlations between the expression levels of the molecules were studied using the Pearson product moment correlation test.

For purposes of the Kaplan-Meier survival analysis, the samples were divided arbitrarily into high and low transcription groups, with the value for the moderate prognostic group as defined by Nottingham prognostic index (NPI) serving as the dividing line. Survival analyses were performed using PSAW18 (SPSS, Inc., Chicago, IL, USA).

\section{Results}

Significantly higher mTORC1 mRNA transcript levels were found in the breast cancer specimens compared to the normal glandular tissue $(\mathrm{P}=0.0018)$. The expression of mTORC1 mRNA was demonstrated to increase with increasing NPI (from 53 for NPI1 to 219 for NPI3) and tumour grade, and this difference reached statistical significance when comparing grade 2 with grade 3 ( 37 vs. 159, $\mathrm{P}=0.047$ ). mTORC1 expression was found to be higher in ductal tumours compared with non-ductal tumours $(\mathrm{P}=0.0014)$. The patients who developed recurrent disease or died from breast cancer had higher expression levels of mTORC1 than those who had been disease-free after a median follow-up period of 10 years $(\mathrm{P}=0.17)$. Higher expression levels were significantly associated with worse overall survival ( $\mathrm{P}=0.01$; Table III, Figs. 1 and 2$)$.

The mTORC2 core protein Rictor mRNA expression showed an opposing trend to mTORC1 with higher levels being found in normal breast tissue, lower NPI stage (NPI1 vs. 2, $\mathrm{P}=0.03$ ) and lower tumour grade (grade 1 vs. $3, \mathrm{P}=0.01$ and grade 2 vs. $3, \mathrm{P}=0.03$ ). Patients with higher Rictor expression had a significantly better disease-free $(\mathrm{P}=0.048)$ and overall $(\mathrm{P}=0.037)$ survival (Table IV, Figs. 3 and 4).

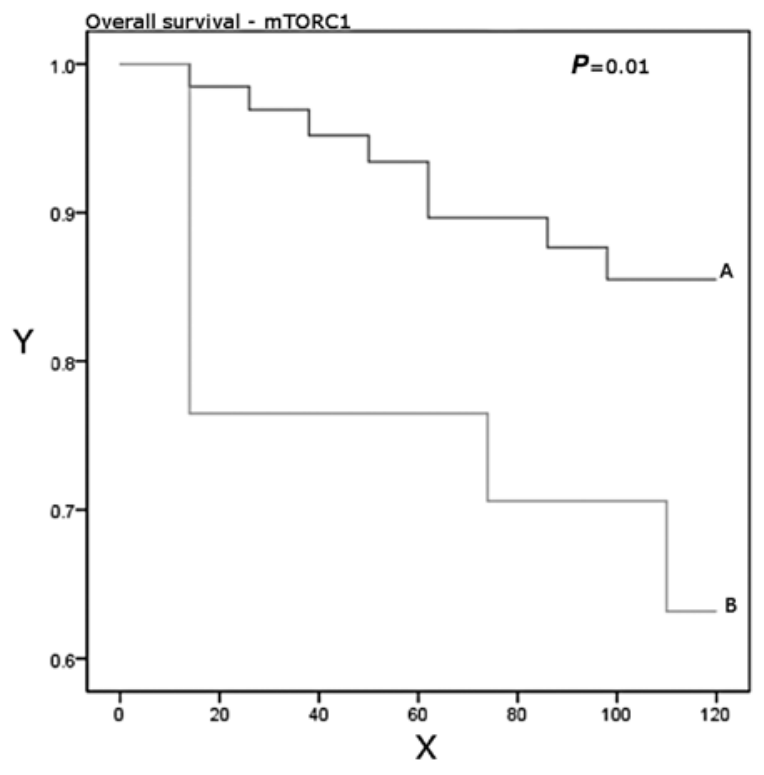

Figure 2. Overall survival curve for mTORC1. Curve A, lower transcription group as determined by moderate prognostic group as defined by NPI serving as the dividing line; Curve B, higher transcription group as determined by moderate prognostic group as defined by NPI serving as the dividing line; $\mathrm{X}$-axis, follow-up in months; Y-axis, cumulative survival.

Table III. Comparison of mTOR mRNA expression levels in subgroups within the cohort.

Patient and tumour

characteristics mTOR, mean (SD) P-value

Tumour grade

1 vs. 2

1 vs. 3

$59(123)$ vs. $36.9(98.9)$
$59(123)$ vs. $139(349)$
$36.9(98.9)$ vs. $139(349)$

0.50

2 vs. 3

0.16

NPI

1 vs. 2

53 (127) vs. 102 (306)

0.047

1 vs. 3

53 (127) vs. 219 (437)

0.36

2 vs. 3

102 (306) vs. 219 (437)

0.17

0.36

TNM

1 vs. 2

$61(134)$ vs. $130(383)$

0.31

1 vs. 3

61 (134) vs. 93 (218)

0.74

1 vs. 4

61 (134) vs. 36.3 (55.7)

0.48

2 vs. 3

130 (383) vs. 93 (218)

0.75

2 vs. 4

130 (383) vs. 36.3 (55.7)

0.19

3 vs. 4

93 (218) vs. 36.3 (55.7)

0.57

Survival

DF vs. LR $63(153)$ vs. $45.5(82.7) \quad 0.63$

DF vs. DR $63(153)$ vs. $20.5(31.7) \quad 0.068$

DF vs. D 63 (153) vs. $324(585) \quad 0.12$

DF vs. LR/DR/D $63(153)$ vs. $191(449) \quad 0.17$

Mean mTOR mRNA expression levels (copy number) in a cohort of 150 breast cancer patients; a comparison between subgroups with different tumour grade, Nottingham prognostic index (NPI), TNM stage and clinical outcome. SD, standard deviation; DF, disease-free survival; LR, local disease recurrence; DR, distant disease recurrence; $\mathrm{D}$, death from breast cancer. 


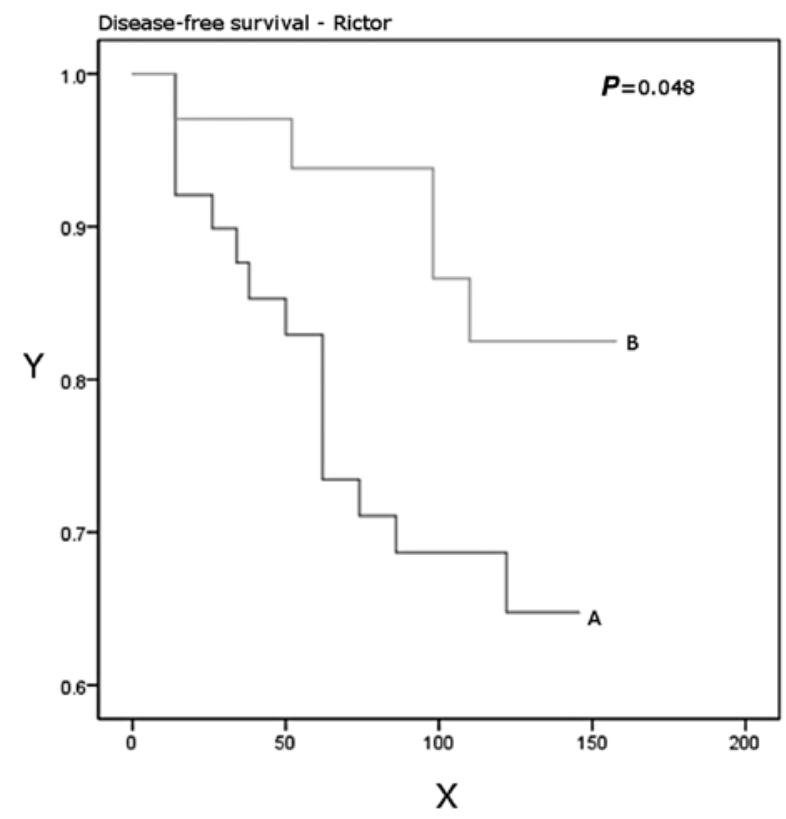

Figure 3. Disease-free survival curve for Rictor. Curve A, lower transcription group as determined by moderate prognostic group as defined by NPI serving as the dividing line; Curve B, higher transcription group as determined by moderate prognostic group as defined by NPI serving as the dividing line; $\mathrm{X}$-axis, follow-up in months; Y-axis, cumulative survival.

Table IV. Comparison of Rictor mRNA expression levels in subgroups within the cohort.

\begin{tabular}{lcc}
\hline $\begin{array}{l}\text { Patient and tumour } \\
\text { characteristics }\end{array}$ & 95\% confidence interval & P-value \\
\hline $\begin{array}{l}\text { Tumour grade } \\
1 \text { vs. } 2\end{array}$ & -3.1 to 11.5 & 0.54 \\
1 vs. 3 & 0.3 to 46.1 & 0.01 \\
2 vs. 3 & -0.2 to 4.7 & 0.039 \\
NPI & & \\
1 vs. 2 & 0.1 to 8.8 & 0.03 \\
1 vs. 3 & -0.6 to 9.0 & 0.16 \\
2 vs. 3 & -2.5 to 1.1 & 0.73 \\
TNM & & \\
1 vs. 2 & -0.7 to 3.7 & 0.622 \\
1 vs. 3 & -8.5 to 24.2 & 0.93 \\
1 vs. 4 & -105.9 to 120.4 & 0.74 \\
2 vs. 3 & -9.0 to 2.1 & 0.72 \\
2 vs. 4 & -248.9 to 2.6 & 0.32 \\
3 vs. 4 & -249.4 to 65.1 & 0.85 \\
Survival & & \\
DF vs. LR & -3.3 to 6.4 & 0.56 \\
DF vs. DR & -98.9 to 13.3 & 0.68 \\
DF vs. D & -2.0 to 1.8 & 0.69 \\
\hline
\end{tabular}

Rictor mRNA expression levels (copy number) in a cohort of 150 breast cancer patients; a comparison between subgroups with different tumour grade, Nottingham prognostic index (NPI), TNM stage and clinical outcome. DF, disease-free survival; LR, local disease recurrence; $\mathrm{DR}$, distant disease recurrence; $\mathrm{D}$, death from breast cancer.

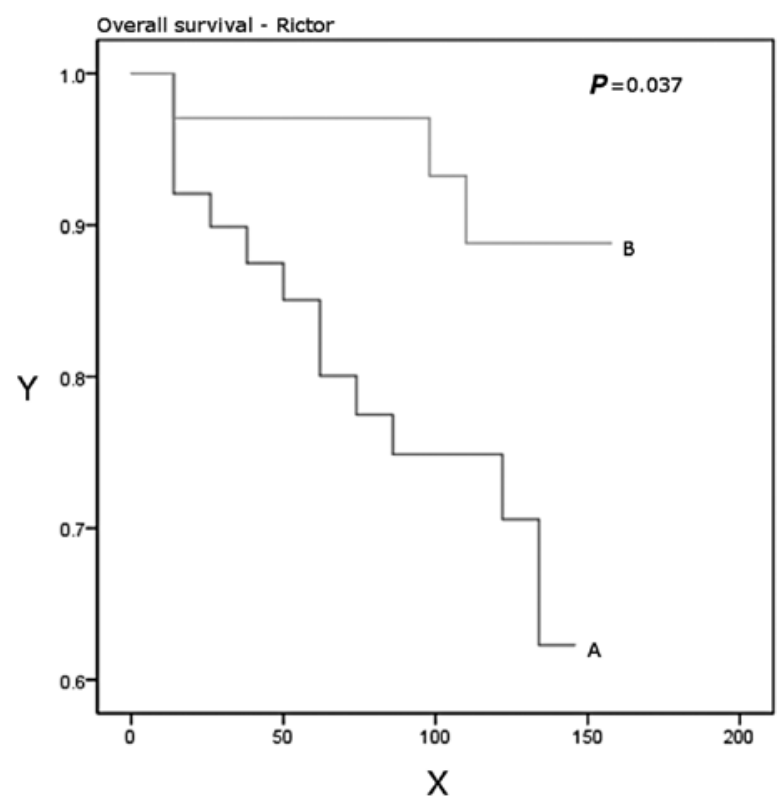

Figure 4. Overall survival curve for Rictor. Curve A, lower transcription group as determined by moderate prognostic group as defined by NPI serving as the dividing line; Curve B, higher transcription group as determined by moderate prognostic group as defined by NPI serving as the dividing line; $\mathrm{X}$-axis, follow-up in months; Y-axis, cumulative survival.

Table V. Comparison of Raptor mRNA expression levels in subgroups within the cohort.

Patient and tumour

characteristics $\quad 95 \%$ confidence interval P-value

Tumour grade

1 vs. 2
1 vs. 3
2 vs. 3
NPI
1 vs. 2
1 vs. 3
2 vs. 3

$-1,144.2$ to 0.0
-946.7 to 0.0
-27.8 to 63.0

0.03

1 vs. 3

0.027

NPI

TNM

1 vs. 2

-0.6 to 118.3

0.8

-26.1 to 457.0

0.42

-29.4 to 42.4

0.33

0.87

1 vs. 3

-225.6 to 1.5

0.46

-0.3 to $1,152.8$

0.21

1 vs. 4

0.0 to $12,201.9$

0.034

2 vs. 3

2 vs. 4

-0.4 to $4,412.4$

0.1076

0.2 to $19,574.1$

0.028

3 vs. 4

-0.01 to 122.43

0.139

Survival

DF vs. LR
DF vs. DR
DF vs. D

-0.0 to $4,873.3$

0.1317

-1973.0 to $12,654.3$

0.5169

-3.5 to 865.7

0.3875

Raptor mRNA expression levels (copy number) in a cohort of 150 breast cancer patients; a comparison between subgroups with different tumour grade, Nottingham prognostic index (NPI), TNM stage and clinical outcome. DF, disease-free survival; LR, local disease recurrence; DR, distant disease recurrence; D, death from breast cancer. 
Table VI. Comparison of Rheb mRNA expression levels in subgroups within the cohort.

\begin{tabular}{lrr}
$\begin{array}{l}\text { Patient and tumour } \\
\text { characteristics }\end{array}$ & $95 \%$ confidence interval & P-value \\
\hline $\begin{array}{l}\text { Tumour grade } \\
1 \text { vs. } 2\end{array}$ & -1044.6 to 0.0 & 0.0127 \\
1 vs. 3 & -0.26 to -0.01 & 0.0078 \\
2 vs. 3 & 0.1 to 12.2 & 0.1768 \\
\hline
\end{tabular}

Rheb mRNA expression levels (copy number) in a cohort of 150 breast cancer patients; a comparison between subgroups with different tumour grade.

The mTORC1 core protein Raptor mRNA expression was higher in the breast cancer specimens, and was found to be directly associated with tumour grade (grade 1 vs. $3, \mathrm{P}=0.027$ ). Paradoxically, it was found to be inversely correlated with TNM staging (TNM1 vs. 4, $\mathrm{P}=0.0343$; Table V). Rheb mRNA expression was directly associated with tumour grade (grade 1 vs. 3, P=0.0078; Table VI). The mTORC1 mRNA showed a highly significant positive correlation with that of hTERT $(\mathrm{r}=0.585, \mathrm{P}<0.00001$; Table VII).

\section{Discussion}

Rapamycin is macrolide produced by Streptomyces hygroscopius. It was initially identified in soil samples taken from the Easter Islands, also known as Rapa Nui (9). It was remarkable for its effect on metabolism and cell growth. During the early 1990s, the studies of the effects of rapamycin on yeasts led to the discovery of the targets of rapamycin (TOR1 and 2). The mammalian targets of rapamycin (mTOR) was described shortly thereafter (10).

Initially, it was found that mTOR formed a multi-protein complex, later termed mTOR complex 1 (mTORC1). Other components of mTORC1 are mammalian lethal with sec-13 protein 8 (mLST8, also known as G $\beta \mathrm{L}$ ), regulatory-associated protein of mammalian target of rapamycin (Raptor) (11), DEP domain containing mTOR-interacting protein (DEPTOR), the Tti1/Tel2 complex, and proline-rich Akt substrate $40 \mathrm{kDa}$ (PRAS40) (10). The activity of mTORC1 is inhibited by rapamycin and other rapamycin analogues (rapalogues). Rapamycin forms a complex with FKBP12, which interacts with mTOR subunit of mTORC1 (12).

mTORC1 has been shown to be affected by various stimuli, including but not limited to hypoxia, nutritional state, stress, growth factors and amino acids. The majority of these stimuli are mediated by a number of effectors, such as protein kinase $B$ (PKB, also known as Akt), extracellular-signal-regulated kinase 1/2 (ERK1/2) and ribosomal S6 kinase (RSK1) (10). Typically, these enzymes phosphorylate the tuberous sclerosis complex 1 and 2 (TSC1/2, also known as hamartin and tuberin, respectively) (13). This inactivates $\mathrm{TSC} 1 / 2$, which when active suppresses the activity of Ras homolog enriched in brain (Rheb). In such conditions, Rheb activates mTORC1 (14). The canonical Wnt pathway interacts with Rheb, as does the insulin/PI3K/Akt pathway $(15,16)$. Alternatively, several upstream components may interact directly with Rheb. This is true with regards to mitogen-activated protein kinase (MAPK), which mediates stimuli from p38 (17). PKB/Akt also to a certain extent, acts directly upon Rheb. In turn, mTORC1 regulates lipid metabolism, glycolysis, protein synthesis, cell proliferation and autophagy $(10,18)$.

In addition, upstream and downstream components of the mTOR pathway have been implicated in carcinogenesis. This has marked mTOR as a potential therapeutic target in cancer treatment (19). Rapalogues have been clinically proven in the treatment of renal cell carcinoma. Their limited side-effect profile makes their particularly attractive $(4,5)$. However, rapalogues have had limited success as single agents in the case of other types of cancers. Considerable efforts have been made to identify potential markers of rapamycin sensitivity $(7,20)$.

A less well described complex 2 (mTORC2) was also later identified. Similar to mTORC1, it is composed of the mTOR, mLST8, DEPTOR and Tti1/Tel2 complex (10). In addition it also contains rapamycin-insensitive companion of mTOR

Table VII. Pearson moment-product correlations.

\begin{tabular}{|c|c|c|c|c|}
\hline & Rictor & Raptor & hTERT & Rheb \\
\hline \multicolumn{5}{|l|}{ mTOR } \\
\hline Correlation coefficient & 0.221 & -0.0249 & 0.585 & -0.0294 \\
\hline $\mathrm{P}$-value & 0.0189 & 0.812 & 4.417E-011 & 0.778 \\
\hline \multicolumn{5}{|l|}{ Rictor } \\
\hline Correlation coefficient & & -0.0515 & 0.181 & -0.0639 \\
\hline P-value & & 0.620 & 0.0614 & 0.538 \\
\hline \multicolumn{5}{|l|}{ Raptor } \\
\hline Correlation coefficient & & & 0.0172 & -0.0241 \\
\hline $\mathrm{P}$-value & & & 0.873 & 0.828 \\
\hline \multicolumn{5}{|l|}{ hTERT } \\
\hline Correlation coefficient & & & & -0.0482 \\
\hline P-value & & & & 0.654 \\
\hline
\end{tabular}


(Rictor) (21), mammalian stress-activated map kinase-interacting protein 1 ( $\mathrm{mSin} 1)$, and protein observed with Rictor 1 and 2 (protor1/2) (3). mTORC2 is not sensitive to rapamycin. Our understanding of the role of mTORC 2 in the wider pathway is still evolving. It has been found to have a role in the regulation of cell survival and the cytoskeleton through the stimulation of various kinases, including Akt/PKB. Through $\mathrm{Akt} / \mathrm{PKB}$, it is also thought to inhibit mTORC1 (22).

hTERT is one of the three components of the telomerase complex, the others being the RNA template (hTR), and the associated protein (TEP-1). This complex repairs the sequences on the ends of chromosomes, which are termed telomeres. This function prevents cell senescence, and could be related with cell immortality (23). Increased hTERT activity has been implicated in various neoplastic diseases, including breast carcinoma (24). In endometrial carcinoma, rapamycin has been demonstrated to reduce hTERT mRNA expression (25). On the other hand, in hepatocellular carcinoma, rapamycin has been found to exert a post-transcriptional inhibitory effect on hTERT activity. However, no effect was found on hTERT mRNA expression (26). Evidence for a regulatory role for mTOR on telomerase activity has been found in the case of adult T cell leukaemia (27). hTERT activity has been suggested as a surrogate for rapamycin antineoplastic activity in endometrial carcinoma (25). Our finding of a highly significant positive correlation between mTORC1 and hTERT lends further support to the hypothesis that mTORC1 is an important regulator of hTERT and telomerase.

Our study shows significant correlations between the expression of mTORC1 and parameters of advanced disease in mammary carcinogenesis, including tumour grade and NPI and an inverse relationship to overall survival. This reiterates the potential for a therapeutic role for mTORC1 inhibitors in human breast cancer. The correlation of mTORC1 expression with ductal carcinoma suggests that such therapeutic agents may be more effective in treating patients with invasive ductal carcinoma. Our findings also suggest that mTORC1 may exert its carcinogenic effect through the upregulation of hTERT.

Whilst Raptor's association with worsening tumour grade is consistent with its role in mTORC1, its paradoxical relationship with TNM stage would require further study along with other components of mTORC1.

This is the first study to show an inverse relationship between the Rictor subunit of mTORC2, NPI and tumour grade, as well as demonstrate a significant direct correlation of Rictor expression with disease-free and overall survival. This is suggestive of a more extensive countervailing role of mTORC2 versus mTORC1 than previously postulated.

\section{Acknowledgements}

This study was funded by grants from the Breast Cancer Hope Foundation (London, UK).

\section{References}

1. Shiota C, Woo JT, Lindner J, Shelton KD and Magnuson MA Multiallelic disruption of the rictor gene in mice reveals that mTOR complex 2 is essential for fetal growth and viability. Dev Cell 11: 583-589, 2006.

2. Wu WK, Lee CW, Cho CH, Chan FK, Yu J and Sung JJ: RNA interference targeting raptor inhibits proliferation of gastric cancer cells. Exp Cell Res 317: 1353-1358, 2011.
3. Guertin DA and Sabatini DM: Defining the role of mTOR in cancer. Cancer Cell 12: 9-22, 2007.

4. Hudes G, Carducci M, Tomczak P, et al: Temsirolimus, interferon alfa, or both for advanced renal-cell carcinoma. N Engl J Med 356: 2271-2281, 2007.

5. Houghton PJ: Everolimus. Clin Cancer Res 16: 1368-1372, 2010.

6. Shor B, Zhang WG, Toral-Barza L, et al: A new pharmacologic action of CCI-779 involves FKBP12-independent inhibition of mTOR kinase activity and profound repression of global protein synthesis. Cancer Res 68: 2934-2943, 2008.

7. Noh WC, Mondesire WH, Peng J, et al: Determinants of rapamycin sensitivity in breast cancer cells. Clin Cancer Res 10: 1013-1023, 2004

8. Jiang WG, Watkins G, Lane J, et al: Prognostic value of rho GTPases and rho guanine nucleotide dissociation inhibitors in human breast cancers. Clin Cancer Res 9: 6432-6440, 2003.

9. Vezina C, Kudelski A and Sehgal SN: Rapamycin (AY-22,989), a new antifungal antibiotic. I. Taxonomy of the producing streptomycete and isolation of the active principle. J Antibiot (Tokyo) 28: 721-726, 1975.

10. Laplante M and Sabatini DM: mTOR signaling in growth control and disease. Cell 149: 274-293, 2012.

11. Bjornsti MA and Houghton PJ: The TOR pathway: a target for cancer therapy. Nat Rev Cancer 4: 335-348, 2004.

12. Brown EJ, Albers MW, Shin TB, et al: A mammalian protein targeted by G1-arresting rapamycin-receptor complex. Nature 369: 756-758, 1994.

13. Gao X, Zhang Y, Arrazola P, et al: Tsc tumour suppressor proteins antagonize amino-acid-TOR signalling. Nat Cell Biol 4: 699-704, 2002.

14. Manning BD and Cantley LC: Rheb fills a GAP between TSC and TOR. Trends Biochem Sci 28: 573-576, 2003.

15. Yuen HF, Chan KK, Grills C, et al: Ran is a potential therapeutic target for cancer cells with molecular changes associated with activation of the PI3K/Akt/mTORC1 and Ras/MEK/ERK pathways. Clin Cancer Res 18: 380-391, 2012.

16. Inoki K, Ouyang H, Zhu T, et al: TSC 2 integrates Wnt and energy signals via a coordinated phosphorylation by AMPK and GSK3 to regulate cell growth. Cell 126: 955-968, 2006.

17. Zheng M, Wang $\mathrm{YH}, \mathrm{Wu} \mathrm{XN}$, et al: Inactivation of Rheb by PRAK-mediated phosphorylation is essential for energy-depletion-induced suppression of mTORC1. Nat Cell Biol 13: 263-272, 2011.

18. Toschi A, Lee E, Thompson S, et al: Phospholipase D-mTOR requirement for the Warburg effect in human cancer cells. Cancer Lett 299: 72-79, 2010.

19. Guertin DA and Sabatini DM: The pharmacology of mTOR inhibition. Sci Signal 2: pe24, 2009.

20. Shoji K, Oda K, Kashiyama T, et al: Genotype-dependent efficacy of a dual PI3K/mTOR inhibitor, NVP-BEZ235, and an mTOR inhibitor, RAD001, in endometrial carcinomas. PLoS One 7: e37431, 2012.

21. Sarbassov DD, Ali SM, Kim DH, et al: Rictor, a novel binding partner of mTOR, defines a rapamycin-insensitive and raptorindependent pathway that regulates the cytoskeleton. Curr Biol 14: 1296-1302, 2004.

22. McDonald PC, Oloumi A, Mills J, et al: Rictor and integrinlinked kinase interact and regulate Akt phosphorylation and cancer cell survival. Cancer Res 68: 1618-1624, 2008.

23. Mokbel K: The role of telomerase in breast cancer. Eur J Surg Oncol 26: 509-514, 2000.

24. Elkak A, Mokbel R, Wilson C, Jiang WG, Newbold RF and Mokbel K: hTERT mRNA expression is associated with a poor clinical outcome in human breast cancer. Anticancer Res 26: 4901-4904, 2006.

25. Zhou C, Gehrig PA, Whang YE and Boggess JF: Rapamycin inhibits telomerase activity by decreasing the hTERT mRNA level in endometrial cancer cells. Mol Cancer Ther 2: 789-795, 2003.

26. Bu X, Jia F, Wang W, Guo X, Wu M and Wei L: Coupled down-regulation of mTOR and telomerase activity during fluorouracil-induced apoptosis of hepatocarcinoma cells. BMC Cancer 7: 208, 2007.

27. Yamada O, Ozaki K, Akiyama M and Kawauchi K: JAK-STAT and JAK-PI3K-mTORC1 pathways regulate telomerase transcriptionally and posttranslationally in ATL cells. Mol Cancer Ther 11: 1112-1121, 2012. 